\title{
Performance Improvement of Raman Distributed Temperature System by Using Noise Suppression
}

\author{
Jian $\mathrm{LI}^{1,2}$, Yunting $\mathrm{LI}^{1,2}$, Mingjiang ZHANG ${ }^{1,2^{*}}$, Yi LIU ${ }^{1,2}$, Jianzhong ZHANG ${ }^{1,2}$, \\ Baoqiang YAN, Dong WANG ${ }^{1,2}$, and Baoquan $\mathrm{JIN}^{1,2}$
}

\begin{abstract}
${ }^{1}$ Key Laboratory of Advanced Transducers and Intelligent Control System of Ministry of Education, Taiyuan University of Technology, Taiyuan, 030024, China

${ }^{2}$ College of Physics and Optoelectronics, Institute of Optoelectronic Engineering, Taiyuan University of Technology, Taiyuan, 030024, China
\end{abstract}

*Corresponding author: Mingjiang ZHANG E-mail: zhangmingjiang@tyut.edu.cn

\begin{abstract}
In Raman distributed temperature system, the key factor for performance improvement is noise suppression, which seriously affects the sensing distance and temperature accuracy. Therefore, we propose and experimentally demonstrate dynamic noise difference algorithm and wavelet transform modulus maximum (WTMM) to de-noising Raman anti-Stokes signal. Experimental results show that the sensing distance can increase from $3 \mathrm{~km}$ to $11.5 \mathrm{~km}$ and the temperature accuracy increases to $1.58{ }^{\circ} \mathrm{C}$ at the sensing distance of $10.4 \mathrm{~km}$.
\end{abstract}

Keywords: Optical fiber sensors; Raman scattering; noise suppression; wavelet transform modulus maximum

Citation: Jian LI, Yunting LI, Mingjiang ZHANG, Yi LIU, Jianzhong ZHANG, Baoqiang YAN, et al., "Performance Improvement of Raman Distributed Temperature System by Using Noise Suppression," Photonic Sensors, 2018, 8(2): 103-113.

\section{Introduction}

Since temperature was demodulated successfully by Raman backscattering over an optical fiber in 1985 [1], the Raman distributed temperature system (RDTS) has attracted interest for decades. And the system has been widely used in the online monitoring system of underground gas pipes [2], fault diagnosis of smart grid [3], and large nuclear infrastructures [4]. There are three demodulation methods in RDTS at present: (1) Rayleigh scattering demodulates Raman anti-Stokes scattering [5], (2) Raman Stokes scattering demodulates Raman anti-Stokes scattering [6-7], and (3) Raman anti-Stokes scattering demodulates itself [8]. In the method based on Rayleigh scattering demodulating
Raman anti-Stokes scattering, the ratio of the intensity of Rayleigh scattering and Raman anti-Stokes scattering is used to demodulate the temperature. Another, the ratio of the intensity of Raman Stokes scattering and Raman anti-Stokes scattering is used to demodulate the temperature in the method of Raman Stokes scattering demodulating Raman anti-Stokes scattering. The two demodulation ways are called as dual-demodulation method, and both need two avalanche photodiodes (APD) and two amplifiers, which means higher cost and larger measurement error caused by the modal dispersion between the two different backscattering lights. Although a method by correcting the speed of the two scattering lights has been proposed to eliminate the

Received: 27 October 2017 / Revised: 7 November 2017

(C) The Author(s) 2017. This article is published with open access at Springerlink.com

DOI: $10.1007 / \mathrm{s} 13320-017-0474-5$

Article type: Regular 
measurement error [9], the speed of the two scattering lights is hard to measure. Raman anti-Stokes scattering self-demodulation method uses anti-Stokes scattering to demodulate anti-Stokes scattering, which averts the effect of the modal dispersion between the two backscatterings in principle and just needs one APD and one amplifier. In addition, self-demodulation avoids a way of noise compared with the dual-demodulation and improves the signal to noise ratio, so the method attracts lots of attention. This auto-correction method utilizes reflected anti-Stokes Raman scattering [10] and a double-ended distributed temperature sensor [11].

The wavelet transform modulus maximum (WTMM) method inherits the advantages of wavelet transform, which not only can interpret signals in both time and frequency domains simultaneously [12-13], but also can save the measurement time [14-16]. Moreover, it is available to capture singular points of signal when the signal has low signal to noise ratio (SNR) and has been demonstrated in dual-demodulation RDTS based on Raman Stokes scattering demodulating Raman anti-Stokes scattering [17].

In this paper, we introduce the WTMM method to self-demodulation RDTS to suppress the noise. Furthermore, we propose a dynamic noise difference algorithm to suppress the ground noise of optical receiver, which utilizes the average value of dark current noise floor behind Fresnel reflection to calculate the dynamic ground noise. By combining the dynamic noise difference algorithm with the WTMM method in self-demodulation RDTS, not only the influence of modal dispersion for temperature accuracy is eliminated, but also the sensing distance and temperature accuracy are improved in the experiment. In addition, it will not affect the system measurement time.

\section{Principle}

\subsection{Self-demodulation method}

The intensity of anti-Stokes light can be expressed as

$$
I(T)=\mathrm{CMI}_{0}\left[\exp \left(\frac{\mathrm{h} \Delta v}{\mathrm{k} T}\right)-1\right]^{-1} \exp \left(-\alpha L-\alpha_{a} L\right)
$$

where $\mathrm{C}$ is a constant value determined by central wavelength of anti-Stokes light, $M$ is the amplification factor of optical receiver, $I_{0}$ is the input intensity, $\mathrm{h}$ is Planck's constant, $\Delta v$ is the Raman frequency shift, $\mathrm{k}$ is Boltzmann's constant, $T$ is the measurement temperature where back scattering is happened, $\alpha$ and $\alpha_{a}$ are the attenuation coefficient of input light and anti-Stokes light, respectively, and $L$ is the position where back scattering happens.

In self-demodulation RDTS, temperature is measured by the intensity ratio $[R(T)]$, between anti-Stokes signal $\left[I\left(T_{0}\right)\right]$ of the reference and the measured anti-Stokes $[I(T)]$, which is expressed as

$$
R(T)=\frac{I(T)}{I\left(T_{0}\right)}=\frac{\exp \left(\frac{\mathrm{h} \Delta v}{\mathrm{k} T_{0}}-1\right)}{\exp \left(\frac{\mathrm{h} \Delta v}{\mathrm{k} T}\right)-1}
$$

where $T$ is the measurement temperature, and $T_{0}$ is the temperature along the fiber under test (FUT) and set as reference temperature.

Actually, the power of the input source and the amplification factor of the amplifier change as the time goes by. And they will influence the measurement results. So, a changed power factor $\Delta I$ and a changed amplification factor $\Delta M$ are introduced to $R(T)$, which is shown as follows:

$$
\frac{I(T)}{I\left(T_{0}\right)}=\frac{(M+\Delta M)\left(I_{0}+\Delta I\right)\left[\exp \left(\frac{\mathrm{h} \Delta v}{\mathrm{k} T_{0}}\right)-1\right]}{M I_{0} \exp \left(\frac{\mathrm{h} \Delta v}{\mathrm{k} T}\right)-1}
$$

In order to eliminate the influence of the change of amplification factor and the input power on temperature measurement, an invariable length optical fiber whose temperature is maintained at a fixed value is introduced as correction fiber. The correction fiber is in front of fiber under test (FUT), so the amplification factor and the input power of 
the correction are the same with the FUT both in measured and reference intensities. Thus (3) can be modified as

$$
\begin{gathered}
\frac{I(T) / I\left(T_{0}\right)}{I\left(T_{c}\right) / I\left(T_{c 0}\right)}= \\
\frac{\left[\exp \left(\frac{\mathrm{h} \Delta v}{\mathrm{k} T_{0}}\right)-1\right]\left[\exp \left(\frac{\mathrm{h} \Delta v}{\mathrm{k} T_{c}}\right)-1\right]}{\left[\exp \left(\frac{\mathrm{h} \Delta v}{\mathrm{k} T}\right)-1\right]\left[\exp \left(\frac{\mathrm{h} \Delta v}{\mathrm{k} T_{c 0}}\right)-1\right]}
\end{gathered}
$$

where $T_{c}$ and $T_{c 0}$ stand for the measured and reference temperatures of correction fiber, respectively. And the value of $T_{c}$ is equal to the value of $T_{c 0}$. Using (4), the temperature can be demodulated accurately. The influence of the changes of input power and amplification factor on temperature demodulation resolution also can be eliminated effectively.

\subsection{Dynamic difference noise algorithm}

In RDTS, the Raman signal is submerged in noise which is caused by the optical receiver and data acquisition card. The optical receiver based on APD plays the most important role in the RDTS noise source.

The optical receiver consists of the APD and amplifier. The noise of amplifier is mainly caused by white noise and $1 / f$ noise, the white noise is influenced by temperature, and the $1 / f$ noise is related to frequency. And the ground noise current $\left(I_{N}\right)$ of APD consists of the dark current $\left(I_{D B}\right)$, surface leakage current $\left(I_{D S}\right)$ of APD, and thermal noise current $\left(I_{T}\right)$ of photo detector, which can be expressed as

$$
\begin{gathered}
I_{N}^{2}=I_{D B}^{2}+I_{D S}^{2}+I_{T}^{2}= \\
2 q I_{D} M^{2} F(M) B_{e}+2 q I_{L} B_{e}+\frac{4 k T_{r}}{R_{L}} B_{e}
\end{gathered}
$$

where $q$ is the electronic charge, $I_{D}$ and $I_{L}$ are the dark current and surface leakage current without amplification, respectively, $M$ is the multiplication factor, $F(M)$ is the noise figure which is determined by the characteristics of random noise when avalanche happens, $B_{e}$ is the bandwidth of optical receiver, $\mathrm{k}$ is Boltzmann's constant, $T_{r}$ is the operating temperature of receiver, and $R_{L}$ is the load resistance.

As shown in (5), the value of ground noise current of APD is determined by multiplication factor, noise figure, and operating temperature. Actually, the change in noise figure can be neglected, and the multiplication factor is affected by operating temperature and bias voltage. And the noise of amplifier is also influenced by temperature. So the ground noise changes as the operating temperature and bias voltage change. Usually, researchers consider the operating temperature and voltage are stationary, but actually the temperature and voltage are changing, which limits the sensing distance because of the weak Raman anti-Stokes signal. So a dynamic noise difference algorithm using the average value of dark current noise floor behind Fresnel reflection as dynamic ground noise is proposed. Figure 1 explains this method.

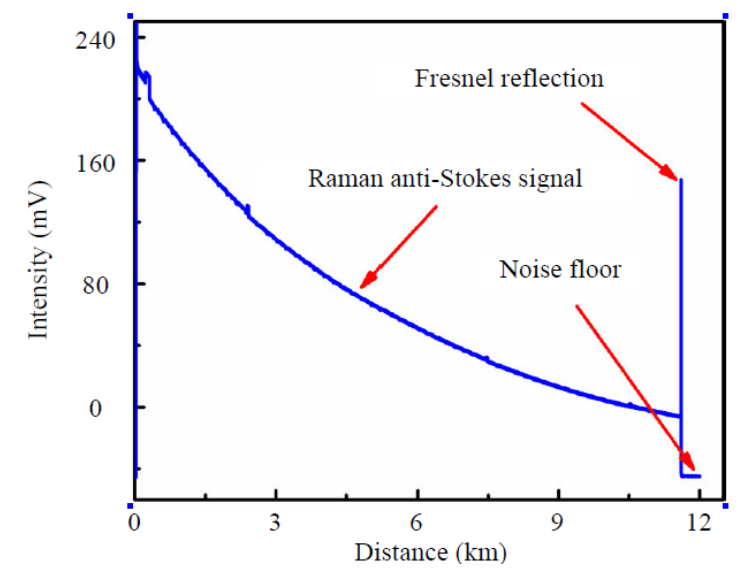

Fig. 1 Raman anti-Stokes backscattering intensity.

Figure 1 shows the Raman anti-Stokes backscattering intensity after 10000-time averages, which includes Raman anti-Stokes signal in the front of Fresnel reflection and noise floor behind Fresnel reflection. The average value of noise floor is used as dynamic ground noise, where the dynamic ground noise will change in different measurement times, and the value just uses one time to calculate the actual intensity of anti-stokes light of this 
measurement time. The dynamic noise difference algorithm is used to obtain the actual intensity of Raman anti-Stokes backscattering, and the value of the actual intensity of Raman anti-Stokes backscattering is calculated by subtracting dynamic ground noise from Raman anti-Stokes signal.

\subsection{Wavelet transform and modulus maximum de-noising}

The wavelet function families are generated from a single base wavelet function $[\varphi(t)]$ dilated and translated by a scale factor $a$ and a location factor $b$. A continuous signal $f(t)^{\text {'s }} \mathrm{s}$ wavelet transform can be express as follows:

$$
W_{f}(a, b)=|\alpha|^{-0.5} \int f(t) \varphi\left(\frac{t-b}{\alpha}\right) d t .
$$

And when the Fourier transform $\varphi(\omega)$ of single base wavelet $\varphi(t)$ conforms to the allowable condition $\left(C_{\varphi}=\int_{0}^{\infty} \frac{|\varphi(\omega)|^{2}}{\omega} d \omega \leq \infty\right)$, the continuous signal $f(t)$ can be expressed as follows:

$$
f(t)=\frac{1}{C_{\varphi}} \int \frac{d a}{a^{2}} \int W_{f}(a, b) \varphi_{a, b}(t) d b .
$$

Then the wavelet function families are discretized, meaning $a=a_{0}^{m}$ and $b=n a_{0}^{m} b_{0}$. And normally $a_{0}=2, b_{0}=1$. The wavelet function families of discrete wavelet transform can be changed into follows:

$$
\varphi_{m, n}(t)=2^{-0.5 m} \varphi\left(2^{m} t-n\right), \quad m, n \in z
$$

Based on (7), a method of multi-resolution analysis (MRA) is proposed by Mallet; the method unifies the construction of base wavelet, and puts forward a fast wavelet decomposition and reconstruction method which is widely used recently. The core of this method is decomposing the signal into detailed high frequency coefficients and approximate low-frequency coefficients, and reconstructing the signal by a filter which is orthogonal with the decomposition filter.

The WTMM is one of the fast wavelet decomposition and reconstruction methods. According to the relationship between Lipschitz exponents $L$ and the scale factor $j$, the noise is reduced in RDTS. The theory [17] can be written as

$$
\left|W_{f}(j, k)\right| \leq \mathrm{c}_{1} j^{L}
$$

where $\mathrm{c}_{1}$ is a constant, and $j$ is the decomposition scale. For the signal, $L>0$, which means the value of the WTMM will increase with an increase in $j$; for the white noise, $L<0$, which will cause the value of the WTMM decrease along with an increase in $j$. According to the characteristics of the signal and noise, WTMM can distinguish the signal and noise effectively.

In our work, the orthogonal wavelet function "sym6" is used because of the characteristics of least asymmetry, regularity, and similarity to signal. The least asymmetry means the method can avoid phase distortion, regularity indicates the de-noised signal can be smoother, and similarity to signal represents signal can be extracted more effectively. The scale of wavelet transform is " 5 " when the SNR of reconstructed detailed coefficient [18] and the results whether the reconstructed signal is distorted are considered. The process of WTMM in our work is shown as follows:

(1) Decompose the original signal by the function "wavedec";

(2) Obtain the reconstructed detailed coefficient and approximate coefficient by the function "wrcoef";

(3) Utilize different threshold values to eliminate the modulus maximum of reconstructed detailed coefficient at different scales, respectively. If the value of modulus maximum is less than threshold, the modulus maximum changes into zero, else reserved;

(4) If the symbol of the value stays the same at each decomposition scale, the rest modulus maximum remains unchanged, else set the value into zero;

(5) Reconstruct the signal based on the reconstructed approximate coefficient and the detailed coefficient after Steps (3) and (4) by the function "waverec". 


\section{Experimental setup}

Figure 2 shows our experimental setup of selfdemodulation method system. The setup is composed of the Raman system, a thermostat (Th), a temperature-controlled chamber (TCC), and multimode fiber (MMF). The Raman system includes a pulsed laser (Laser: XUFENG photoelectric, 1150503), a wavelength division multiplexer (WDM: XUFENG photoelectric, 1105013), an avalanche photodiode \& an amplifier (APD \& AMP: Fby photoelectric, DTS1550-
DA-MM) module, and a data acquisition card (DAC: JEMETECH, JEME-AD 1601). The MMF includes the fiber under test (FUT) and correction optical fiber. The correction optical fiber at $180 \mathrm{~m}-260 \mathrm{~m}$ is put into a thermostat whose temperature is controlled in $26^{\circ} \mathrm{C}$. The bandwidth of APD \& Amp module is $80 \mathrm{MHz}$, and the DAC with $100 \mathrm{MHz}$ bandwidth is triggered synchronously by the synchronizing signal of the pulsed laser. The prototype of RDTS and platform based on LabVIEW are shown in Figs. 3 and 4.

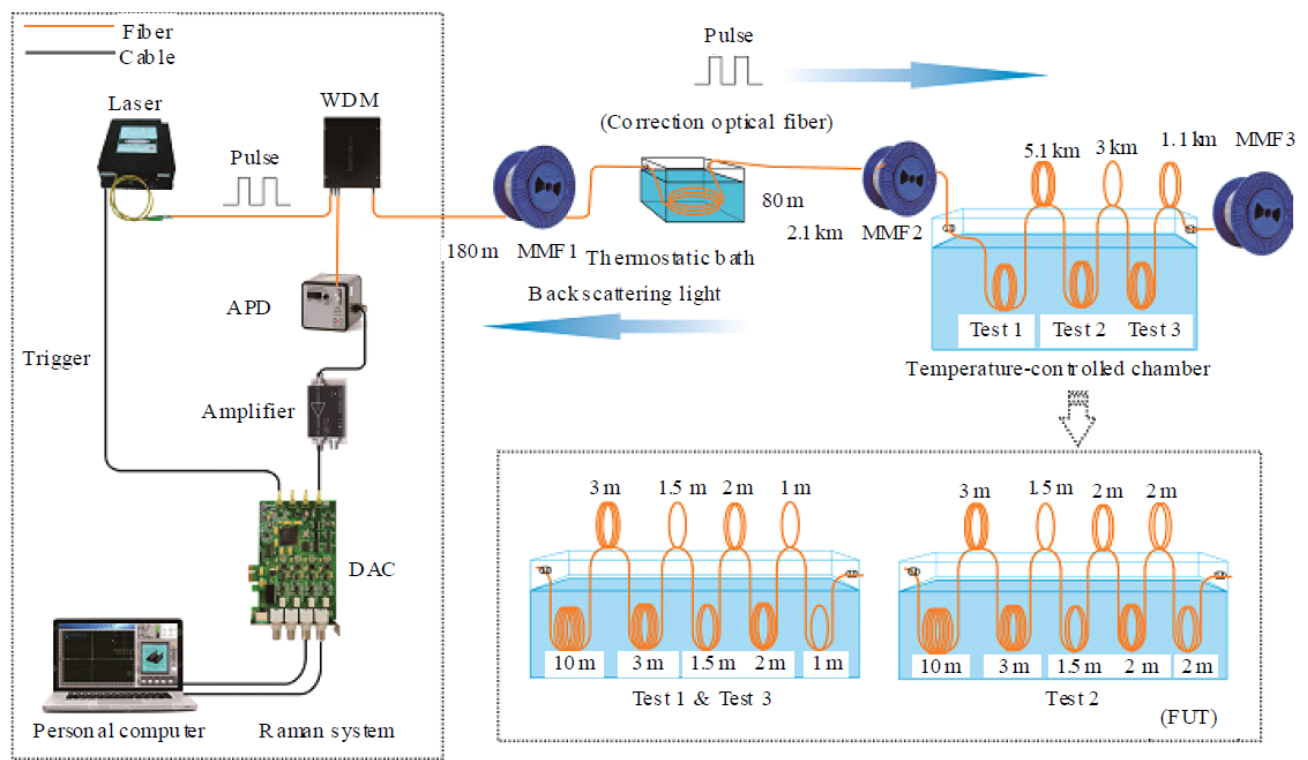

Fig. 2 Experimental setup of RDTS based on self-demodulation method.

In the experiment, a pulsed laser with 10-ns pulse width at $1550 \mathrm{~nm}$ is used as the optical source. The pulse is injected into a WDM, then coupled into an $11.5-\mathrm{km}$ multimode sensing fiber. Then the Raman anti-Stokes backscattering (AS) is filtered by the WDM and detected and amplified by the APD \& AMP module. The intensity of the electrical signal is sampled by the data acquisition card (DAC), then the temperature is demodulated by the personal computer (PC). It is worth noting that the repeat frequency of input light is $8 \mathrm{kHz}$ which means that the sensing distance can reach $12.5 \mathrm{~km}$, but actually the fiber is $11.5 \mathrm{~km}$, so the last $1-\mathrm{km}$ sensing distance is used to get the noise floor, then the dynamic ground noise is calculated.
In Fig. 2, the FUT includes three test zones. In Tests 1 and 3, 10-m, 3-m, 1.5-m, 2-m, and 1-m fibers are all put in the TCC, and the spacings of the fiber are $3 \mathrm{~m}, 1.5 \mathrm{~m}, 2 \mathrm{~m}$, and $1 \mathrm{~m}$, respectively. In Test 2,10-m, 3-m, 1.5-m, 2-m, and 2-m fibers are set in the same TCC, and the spacings of the fiber is $3 \mathrm{~m}, 1.5 \mathrm{~m}, 2 \mathrm{~m}$, and $2 \mathrm{~m}$, respectively.

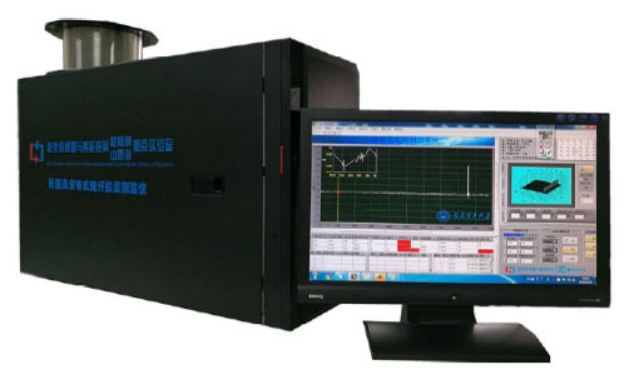

Fig. 3 Prototype of RDTS. 


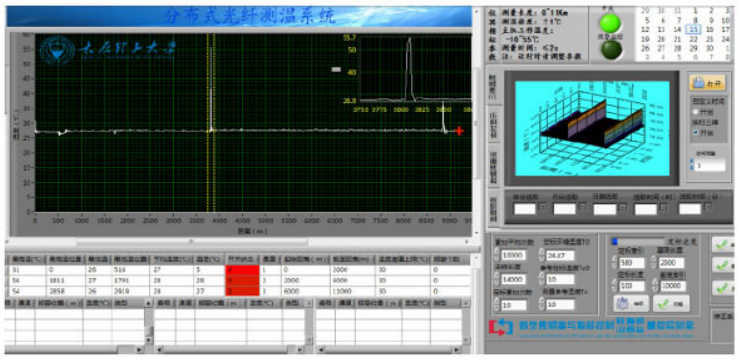

Fig. 4 RDTS platform based on LabVIEW.

\section{Experimental results}

\subsection{Sensing distance lengthened by using dynamic noise difference algorithm}

In the experiment, the three test zones are set in the same TCC (Shanghai Bilon: GDH-3030), the temperature fluctuation is $0.005{ }^{\circ} \mathrm{C}-0.02{ }^{\circ} \mathrm{C}$, and the temperature is measured by Agilent E2308A whose accuracy is $\pm 0.2{ }^{\circ} \mathrm{C}$.

The fluctuation of ground noise of the optical receiver and the influence on measured result are analyzed. The fluctuation of ground noise is shown in Fig. 5. In Fig. 5, the values of the first 57 times are dynamic ground noise, and the last one is calculated by constant noise floor when the measurement is finished and there is no light input. As shown in Fig. 5, the ground noise fluctuates between $-42.18 \mathrm{mV}$ and $-48.81 \mathrm{mV}$. The fluctuation is caused by the changes in operating temperature and bias voltage according to (5).

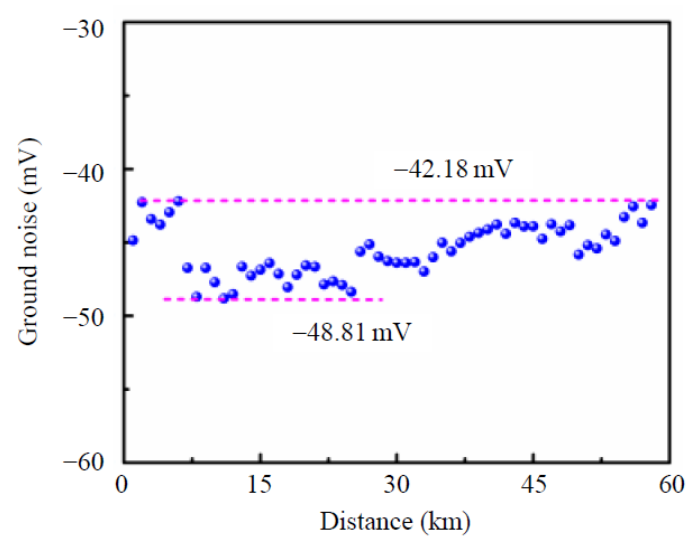

Fig. 5 Fluctuation of ground noise of optical receiver.

In order to prove that the noise difference algorithm can enlarge sensing distance, constant ground noise and dynamic ground noise are used to demodulate temperature when the actual temperature is $40.02{ }^{\circ} \mathrm{C}$, respectively. And the result is shown in Fig. 5. In Fig. 5, the ground noise after measurement whose value is $-42.45 \mathrm{mV}$ is used as constant ground noise; the reference dynamic ground noise is $-44.86 \mathrm{mV}$, and the measurement dynamic ground noise is $-47.19 \mathrm{mV}$.

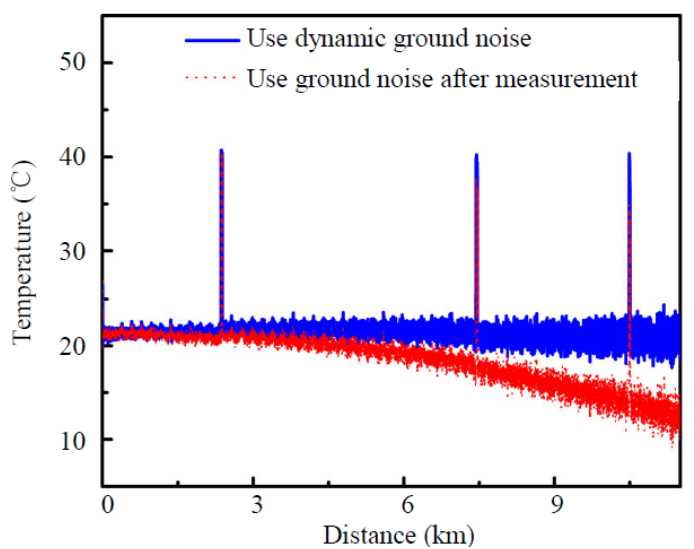

(a)

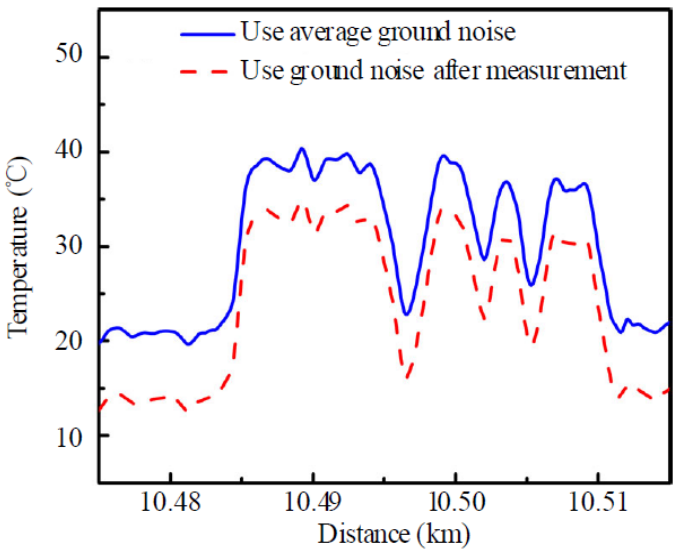

(b)

Fig. 6 Measurement results by using dynamic ground noise and constant ground noise: (a) overall-distribution alone the fiber and (b) at Test 3.

Figure 6(a) shows the measured temperature along the entire fiber, and Fig. 6(b) shows the measured result at Test 3. As shown in Fig. 6(a), the temperature error happens from $3 \mathrm{~km}$ and is enlarging as the sensing distance increases when the constant ground noise is used. In Fig. 6(b), the temperature accuracy optimizes from $6.62{ }^{\circ} \mathrm{C}$ to $1.15^{\circ} \mathrm{C}$ by using dynamic ground noise at Test 3 . In the experiment, it can be considered that the measurement intensity is weaker than actual intensity when constant ground noise is used, so the 
demodulate temperature is less than the actual value, and the demodulate temperature decreases as the intensity of useful signal decreases.

\subsection{Temperature fluctuation decreased by WTMM de-noising}

In the precondition of using the dynamic noise difference algorithm, the comparison between temperature demodulated by original signal and that by the signal after wavelet is shown in Fig. 7. The original signal is averaged 10000 times, the input pulse power is $14.6 \mathrm{~W}$, the decomposition scale of wavelet is " 5 ", and the wavelet function is "sym6". As shown in Fig. 7, the de-noising effect is more and more effective as the sensing distance increases. Especially, in the end of sensing fiber, the

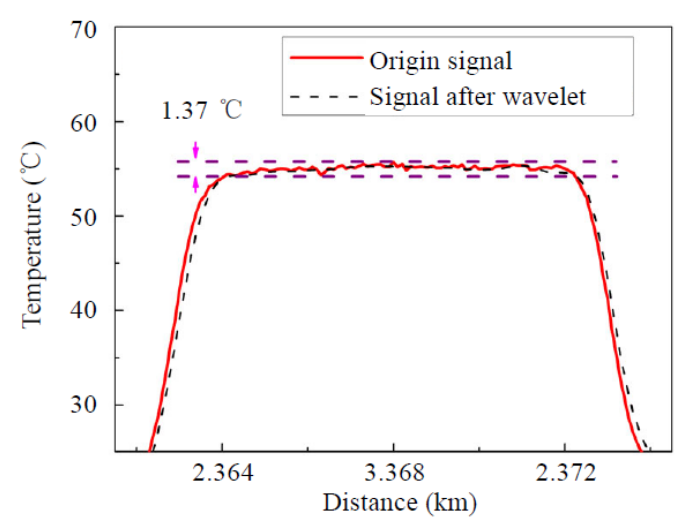

(a)

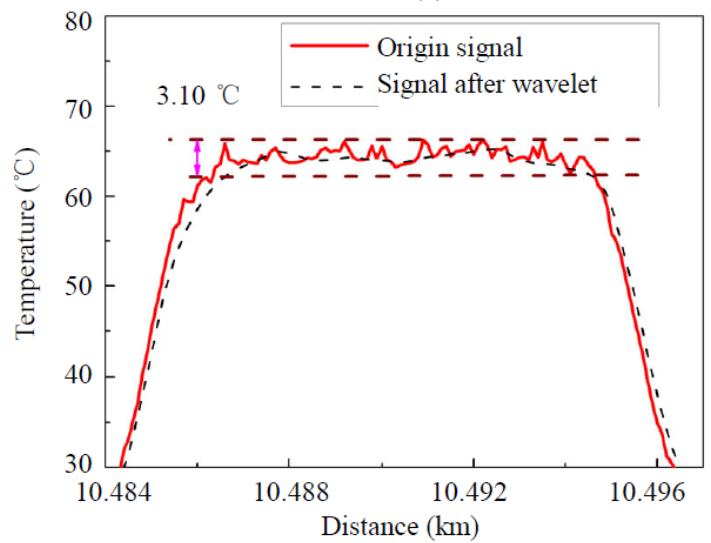

(c) temperature fluctuation changes from $5.98{ }^{\circ} \mathrm{C}$ to $1.23{ }^{\circ} \mathrm{C}$ by using the WTMM method.

The phenomenon above is caused by the signal that is attenuated as the distance increases. In Test 1 , the average value of amplitude of signal is $230.5 \mathrm{mV}$, and changes to $60.8 \mathrm{mV}$ in Test 3 , and then $40 \mathrm{mV}$ at the end of the sensing fiber. Besides, the peak-topeak amplitude of noise changes from $1 \mathrm{mV}$ to $0.1 \mathrm{mV}$ after the WTMM method is used over the sensing fiber. Acording to (4), the intensity ratio of correction optical fiber almost keeps the same, but the ratio of FUT in different distances will be influenced by the noise. So, when the same value of noise is de-noised, the weaker signal results in a higher influence on temperature fluctuation.

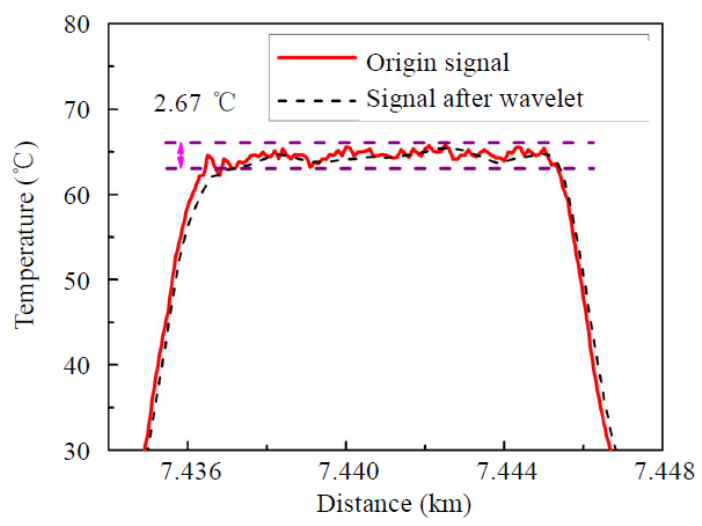

(b)

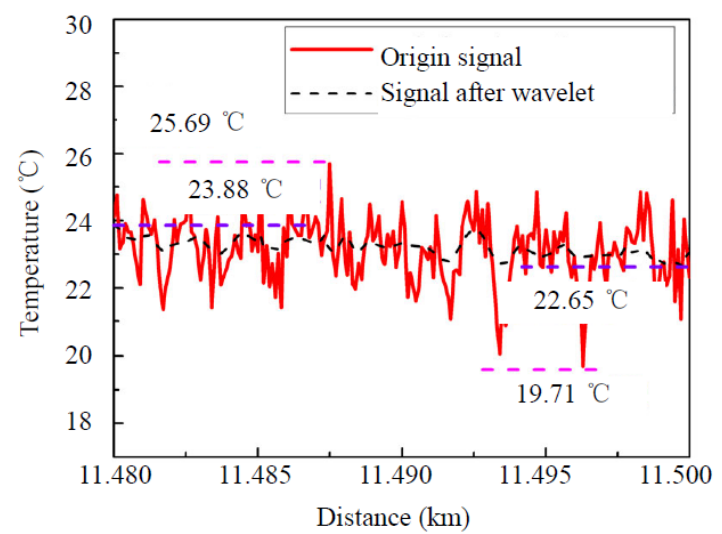

(d)

Fig. 7 Results demodulated by origin signal and the signal after wavelet at different sensing distances at: (a) Test 1, (b) Test 2 , (c) Test 3, and (d) the end of the test fiber under room temperature.

\section{Measurement results}

Figure 8 shows the self-demodulation method measurement results, the temperature accuracy and uncertainty in three different test zones in self-demodulation method by combining dynamic noise difference algorithm with the WTMM method 
when the average room temperature is $22.82{ }^{\circ} \mathrm{C}$, the TCC is adjusted from $35{ }^{\circ} \mathrm{C}$ to $65{ }^{\circ} \mathrm{C}$ and the spacing is about $5{ }^{\circ} \mathrm{C}$. The decomposition scale of

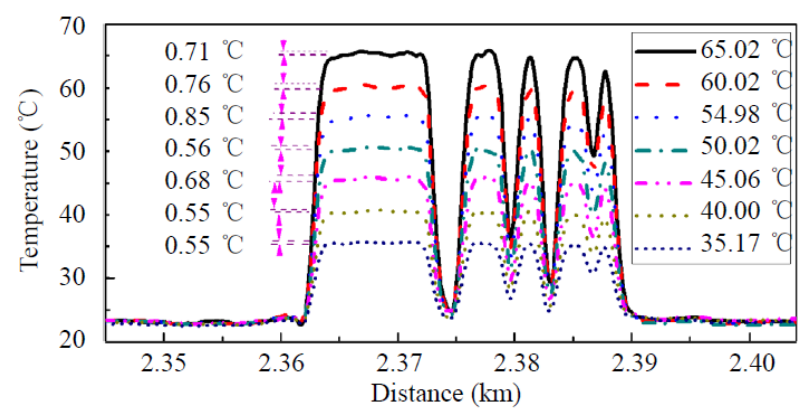

(a1)

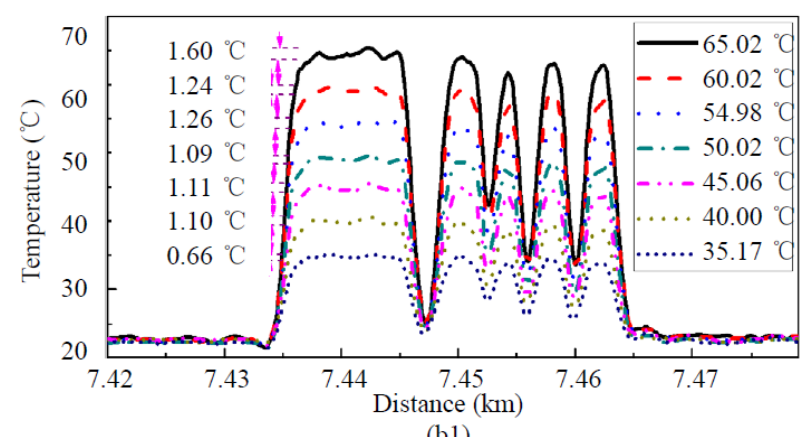

(b1)

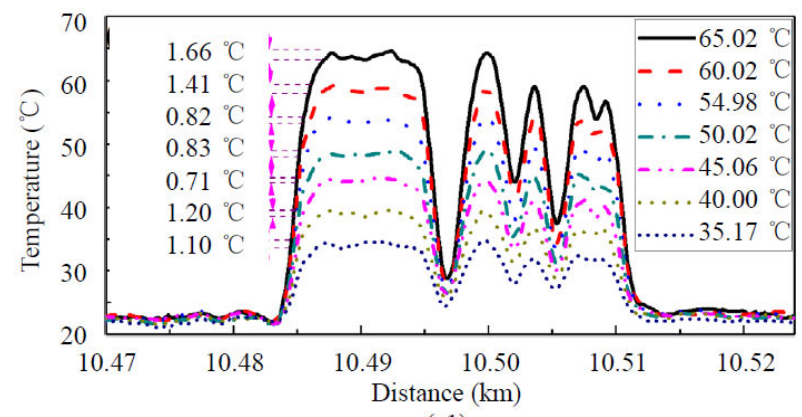

(c1) wavelet is "5" and the wavelet function is "sym6". Figures $8(\mathrm{a}), 8(\mathrm{~b})$, and $8(\mathrm{c})$ stand for the result of Tests 1,2 , and 3 , respectively.

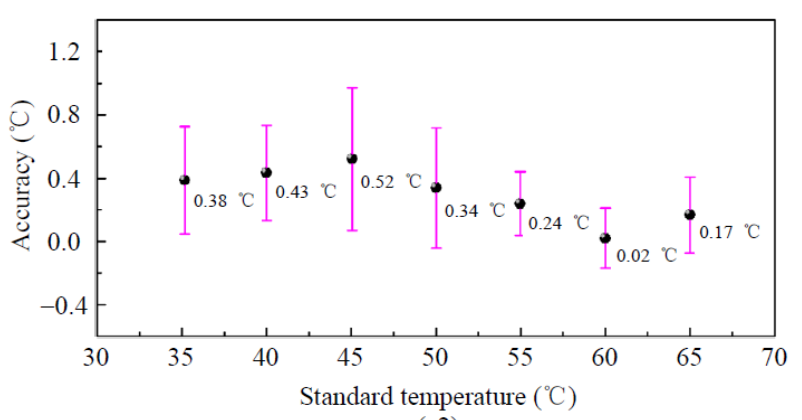

(a2)

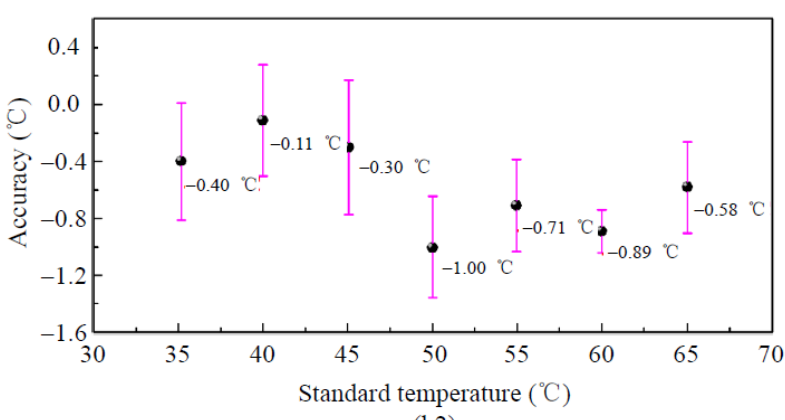

(b2)

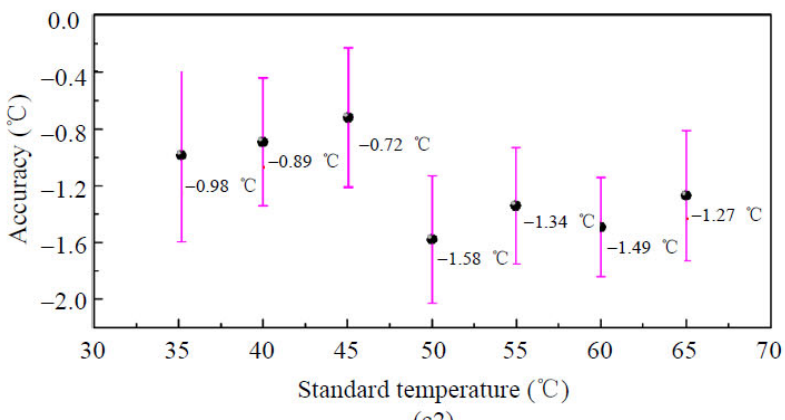

(c2)

Fig. 8 Results of measured temperature in detail and the accuracy and uncertainty of the results at different test zones at: (a) Test 1 , (b) Test 2, and (c) Test 3.

In Figs. 8(a1), 8(b1), and 8(c1), it is obvious that the maximum temperature fluctuation of the three test zones is increasing with the sensing distance increasing. The highest fluctuation appears at Test 3 when the actual temperature is $65.02{ }^{\circ} \mathrm{C}$. This phenomenon is caused by the average amplitude of Raman signal attenuating from $275 \mathrm{mV}$ to $40 \mathrm{mV}$. However, the peak-to-peak of the noise stays the same after cumulative average and WTMM de-noising. The noise remains unchanged, and yet the Raman signal decreases, which must result in the higher temperature fluctuation. The error bar stands for uncertainty of the system which is shown in Figs. 8(a2), 8(b2), and 8(c2). As figures show, the uncertainty is enlarging as the sensing distance increases, which is also caused by the attenuated signal.

The measurement accuracy is also shown in Figs. $8(\mathrm{a} 2), 8(\mathrm{~b} 2)$, and $8(\mathrm{c} 2)$. In the figures, the temperature accuracy is deteriorating as the sensing distance increases. The worst accuracies are $0.52{ }^{\circ} \mathrm{C}$, $-1.00{ }^{\circ} \mathrm{C}$, and $-1.58{ }^{\circ} \mathrm{C}$ at Tests 1,2 , and 3 , respectively. The reason is that WTMM method not only contributes to the signal de-noising, but also 
filters some Raman backscattering signals [14]. According to (4), in the front of the fiber, although some Raman backscattering signals are filtered, the influence on the measurement result does not take into consideration because of the high intensity of the Raman backscattering signals. But at the end of the fiber, the WTMM filters the same useful signal, while the average amplitude of signal attenuates almost $4 \mathrm{~dB}$, so the temperature accuracy is lower than that of the front-end fiber obviously.

The repeatability of the system shown in Table 1 is also analyzed. Three test zones are set in the TCC and the temperature is controlled at $34.87{ }^{\circ} \mathrm{C}$. Then take measurement every 60 minutes. The average values of the five times measurement at three test zones are $35.058{ }^{\circ} \mathrm{C}, 34.116{ }^{\circ} \mathrm{C}$, and $33.786{ }^{\circ} \mathrm{C}$, respectively. The temperature fluctuation of the system at three test zones is $0.90{ }^{\circ} \mathrm{C}, 0.42{ }^{\circ} \mathrm{C}$, and $1.02{ }^{\circ} \mathrm{C}$. This means the repeat ability of the system is well.

Table 1 Repeatability of the system $\left({ }^{\circ} \mathrm{C}\right)$

\begin{tabular}{cccccc}
\hline & 1 & 2 & 3 & 4 & 5 \\
\hline Test 1 & 34.50 & 35.40 & 35.06 & 35.00 & 35.33 \\
Test 2 & 33.94 & 34.21 & 33.89 & 34.18 & 34.36 \\
Test 3 & 33.35 & 34.12 & 33.65 & 34.37 & 33.44 \\
\hline
\end{tabular}

Then we analyze the spatial resolution of the system, and the result is shown in Fig. 9, where the spatial resolution was defined in [19] $(10 \%-90 \%$ response distance to a temperature step). Figures 9(a), 9(b), and 9(c) show the spatial resolution of Tests 1, 2, and 3, respectively, and the relevant spatial resolutions are $1.3 \mathrm{~m}, 1.6 \mathrm{~m}$, and $2.1 \mathrm{~m}$. As shown in Fig. 9, it is obvious that the spatial resolution is deteriorating as the sensing distance increases. This is mainly caused by the degraded SNR and the effect of optical Chromatic dispersion.

The performance parameters of RDTS are as follows:

(1) Temperature accuracy: $\pm 1.58{ }^{\circ} \mathrm{C}$;

(2) Temperature fluctuation: $\pm 0.88{ }^{\circ} \mathrm{C}$;

(3) Sensing distance: $11.5 \mathrm{~km}$;

(4) Response time: $2 \mathrm{~s}$.

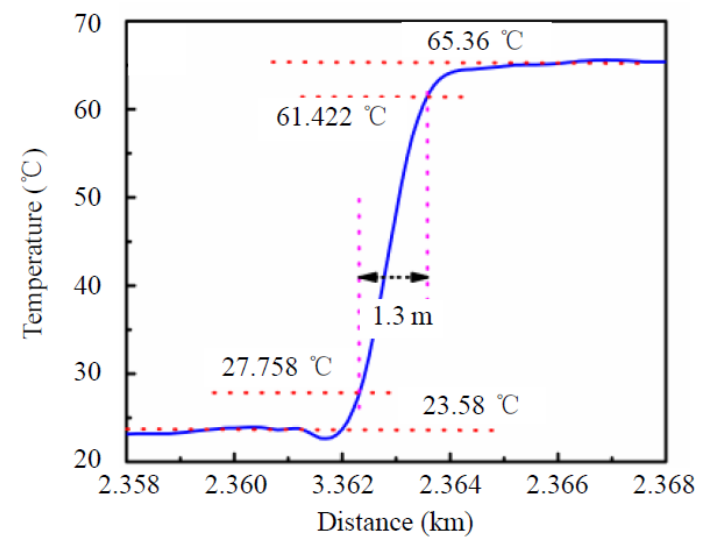

(a)

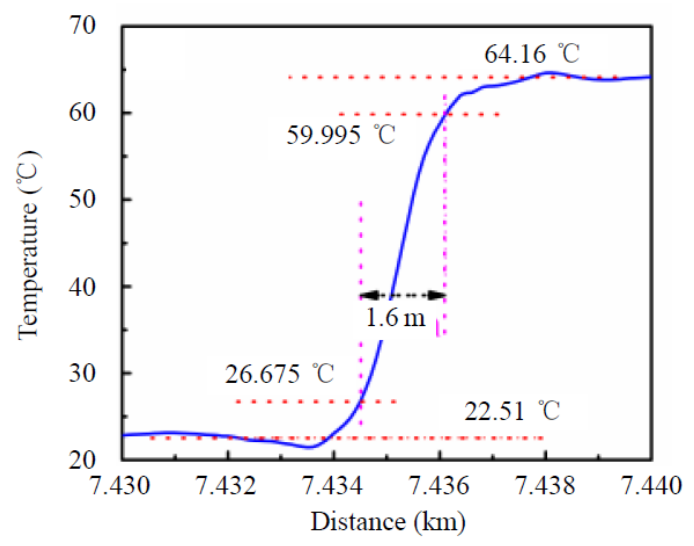

(b)

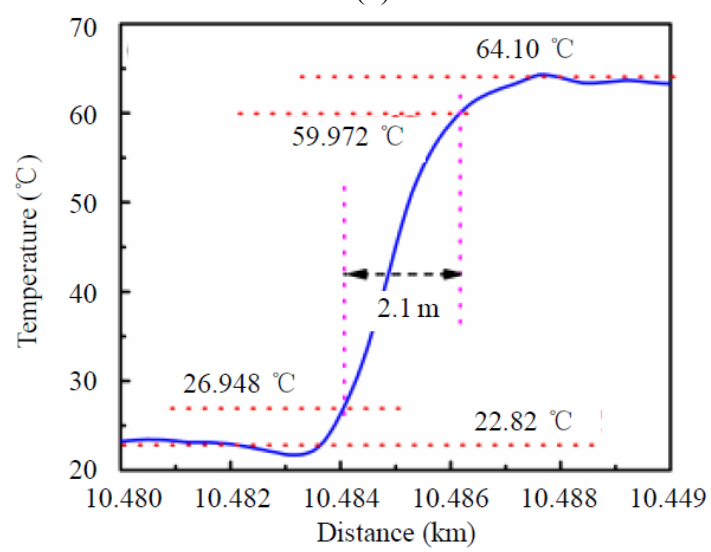

(c)

Fig. 9 Spatial resolution at three different test zones of RDTS at: (a) Test 1, (b) Test 2, and (c) Test 3.

\section{Conclusions}

In this paper, dynamic noise difference algorithm and wavelet transform modulus maximum method are proposed to suppress the noise for self-demodulation Raman distributed temperature system. The system only uses one APD and one amplifier to demodulate the temperature, which eliminates the mode dispersion and saves the cost. In 
experiment, it is demonstrated that the modulus maximum de-noising method can reduce temperature fluctuation, and dynamic noise difference algorithm can enlarge the sensing distance in experiment. As a result, temperature fluctuation of $\pm 0.88{ }^{\circ} \mathrm{C}$ and spatial resolution of $2.1 \mathrm{~m}$ are achieved over an $11.5 \mathrm{~km}$ sensing fiber.

\section{Acknowledgment}

This work is supported by the National Natural Science Foundation of China (NSFC) under Grant Nos. 61377089 and 61527819, by Shanxi Province Natural Science Foundation under Grant No. 2015011049, by Research Project by Shanxi Province Youth Science and Technology Foundation under Grant No. 201601D021069, and by Research Project Supported by Shanxi Scholarship Council of China under Grant No. 2016-036; Key Science and Technology Research Project based on Coal of Shanxi Province (MQ2014-09), by program for the Outstanding Innovative Teams of Higher Learning Institutions of Shanxi, by Program for Sanjin Scholar.

Open Access This article is distributed under the terms of the Creative Commons Attribution 4.0 International License (http://creativecommons.org/ licenses/by/4.0/), which permits unrestricted use, distribution, and reproduction in any medium, provided you give appropriate credit to the original author(s) and the source, provide a link to the Creative Commons license, and indicate if changes were made.

\section{References}

[1] J. P. Dakin, D. J. Pratt, G. W. Bibby, and J. N. Ross, "Distributed optical fibre Raman temperature sensor using a semiconductor light source and detector," Electronics Letters, 1985, 21(13): 569-570.

[2] C. Shen, C. Hu, H. Gong, J. Wang, Y. Jin, and Z Zhang, "Distributed optical fiber temperature sensor," Applied in Underground Coal Gasification System, 2010, 7990: 302-303.

[3] Y. Liu, T. Lei, Z. H. Sun, C. Wang, and T. Y. Li, "Application of distributed optical fiber temperature system in online monitoring and fault diagnosis of smart grid," in Proceeding of Asia-Pacific Power and
Energy Engineering Conference, Jinan, China, 2012, pp. 27-29.

[4] A. F. Fernandez, P. Rodeghiero, B. Brichard, and F. Berghmans, "Radiation-tolerant Raman distributed temperature monitoring system for large nuclear infrastructures," IEEE Transactions on Nuclear Science, 2005, 52(6): 2689-2694.

[5] J. Park, G. Bolognini, D. Lee, P. Kim, P. Cho, and F. D. Pasquale, "Raman-based distributed temperature sensor with simplex coding and link optimization," IEEE Photonics Technology Letters, 2006, 18(17): 1879-1881.

[6] M. Sun, Y. Q. Tang, J. Li, and F. Z. Dong, "Study on spatial resolution improvement of distributed temperature sensor system by linear fitting algorithm," in Proceeding of the Chinese-Societyfor-Optical-Engineering on Applied Optics and Photonics, Beijing, China, 2015, pp. 5-7.

[7] G. D. B. Vazquez, O. E. Martínez, and D. Kunik "Distributed temperature sensing using cyclic pseudorandom sequences," IEEE Sensors Journal, 2017, 17(6): 1686-1691.

[8] M. A. Soto, A. Signorini, T. Nannipieri, S. Bolognini, G. Bolognini, and F. D. Pasquale, "Impact of loss variations on double-ended distributed temperature sensors based on Raman anti-Stokes signal only," Journal of Lightwave Technology, 2012, 30(8): 1215-1222.

[9] W. J. Wang, J. Chang, G. P. Lv, Z. L. Wang, Z. Liu, S. Luo, et al., "Wavelength dispersion analysis on fiber-optic Raman distributed temperature sensor system," Photonic Sensors, 2013, 3(3): 256-261.

[10] D. Hwang, D. J. Yoon, I. B. Kwon, D. C. Seo, and Y. Chung, "Novel auto-correction method in a fiber-optic distributed-temperature sensor using reflected anti-Stokes Raman scattering," Optics Express, 2010, 18(10): 9747-9754.

[11] M. A. Soto, A. Signorini, T. Nannipieri, S. Faralli, and G. Bolognini, "High-performance Raman-based distributed fiber-optic sensing under a loop scheme using anti-stokes light only," IEEE Photonics Technology Letters, 2011, 23(9): 534-536.

[12] Z. Qin, L. Chen, and X. Bao, "Continuous wavelet transform for non-stationary vibration detection with phase-OTDR," Optics Express, 2012, 20(18): 20459-20465.

[13] C. Y. Ma, T. G. Liu, K. Liu, J. F. Jiang, Z. Y. Ding, X. D. Huang, et al., "A Continuous wavelet transform based time delay estimation method for long range fiber interferometric vibration sensor," Journal of Lightwave Technology, 2016, 34(16): 3785-3789.

[14] Y. Hu, W. Q. Mo, K. F. Dong, F. Jin, and J. L. Song, "Using maximum spectrum of continuous wavelet transform for demodulation of an overlapped 
spectrum in a fiber Bragg grating sensor network," Applied Optics, 2016, 55(17): 4670-4675.

[15] X. Feng, X. T. Zhang, C. G. Sun, M. H. Motamedi, and F. Ansari, "Stationary wavelet transform method for distributed detection of damage by fiber-optic sensors," American Society of Civil Engineers, 2014, 140(4): 04013004-1-04013004-11.

[16] M. A. Farahani, M. T. V. Wylie, E. Castillo-Guerra, and B. G. Colpitts, "Reduction in the number of averages required in BOTDA sensors using wavelet denoising techniques," Journal of Lightwave Technology, 2012, 30(8): 1134-1142.

[17] Z. Wang, J. Chang, S. Zhang, S. Luo, C. Jia, and S.
Jiang, "An improved denoising method in RDTS based on wavelet transform modulus maxima," IEEE Sensors Journal, 2015, 15(2): 1061-1067.

[18] Z. L. Wang, J. Chang, S. S. Zhang, S. Luo, C. W. Jia, C. W. Jia, et al., "Application of wavelet transform modulus maxima in Raman distributed temperature sensors," Photonic Sensors, 2014, 4(2): 142-146.

[19] M. A. Soto, T. Nannipieri, A. Signorini, A. Lazzeri, F. Baronti, R. Roncella, et al., "Raman-based distributed temperature sensor with $1 \mathrm{~m}$ spatial resolution over $26 \mathrm{~km}$ SMF using low-repetition-rate cyclic pulse coding," Optics Letters, 2011, 36(13): 2557-2559. 